\title{
Satisfactory Pain Management With Minimal Opioid Use After Arthroscopic Rotator Cuff Repair
}

\author{
Kirsten D. Garvey, M.S., Natalie A. Lowenstein, B.S., Lauren E. Piana, M.D., \\ Kaetlyn R. Arant, B.A., Yuchiao Chang, Ph.D., and Elizabeth G. Matzkin, M.D.
}

\begin{abstract}
Purpose: To evaluate patient use of opioids following arthroscopic rotator cuff repair, including the number of days and number of pills when used in combination with non-opioid medications and to determine whether patients were satisfied with their pain management and if variables such as age, sex, body mass index, duration of symptoms, anticipation of postoperative pain, preoperative opioid consumption, size of the rotator cuff tear, or anxiety/depression affected pain management. Methods: This was a prospective cohort study of 117 prospectively enrolled patients older than the age of 18 years undergoing primary arthroscopic rotator cuff repair. All patients completed preoperative and 2-week postoperative questionnaires to assess their pain and satisfaction with pain management. Univariate and multivariate analyses were performed to evaluate the association of patient characteristics with satisfaction of pain control and amount/duration of opioids postoperatively. Results: Patients required a median of 18 opioid pain pills or 135 morphine milligram equivalents (interquartiles, 6-35 pills) postoperatively over $6.9 \pm 5.1$ days. In total, $65 \%$ of patients took opioid pain medications for 7 days or fewer. On postoperative day 2, patients reported a VAS pain score of $6.6 \pm 2.8$ and at the 2 -week postoperative visit, mean visual analog scale pain score was $3.5 \pm 2.5$. Differences in age, sex, body mass index, duration of symptoms, anticipation of postoperative pain, preoperative 2-item Patient Health Questionnaire, 2-item Pain Self-Efficacy Questionnaire, current opioid use, and surgical characteristics had no effect on, or association with, satisfaction with pain management postoperatively. Conclusions: Following arthroscopic rotator cuff repair, patients can achieve satisfactory pain control using a multimodal approach with a median of 18 opioid pills (range 6-35 pills) over $6.9 \pm 5.1$ days when used in combination with non-opioid pain medications. Overall, 74.4\% of patients were satisfied with their postoperative pain management. Level of Evidence: Level II; Prospective cohort study.
\end{abstract}

$\mathbf{T}$ he growing use and abuse of opioids in the United States is recognized by researchers, government officials, and physicians alike as an epidemic of unforeseen proportions. ${ }^{1}$ According to the Centers for Disease Control and Prevention, it is estimated that in

From the Department of Orthopedic Surgery (K.D.G., N.A.L., E.G.M.) and Orthopedic and Arthritis Center for Outcomes Research (K.R.A., Y.C.), Brigham and Women's Hospital, Harvard Medical School, Boston, Massachusetts; Department of Orthopedic Surgery, Brown Alpert Medical School, Providence, Rhode Island (L.E.P.); and Division of General Internal Medicine, Massachusetts General Hospital, Harvard Medical School, Boston, Massachusetts (Y.C.), U.S.A.

The authors report that they have no conflicts of interest in the authorship and publication of this article. Full ICMJE author disclosure forms are available for this article online, as supplementary material.

Received April 15, 2021; accepted October 9, 2021.

Address correspondence to Elizabeth G. Matzkin, M.D., 75 Francis St., Boston, MA 02155.E-mail: ematzkin@bwh.harvard.edu

(C) 2021 THE AUTHORS. Published by Elsevier Inc. on behalf of the Arthroscopy Association of North America. This is an open access article under the CC BY-NC-ND license (http://creativecommons.org/licenses/by-nc-nd/4.0/).

2666-061X/21555

https://doi.org/10.1016/j.asmr.2021.10.005
2018 , opioids were involved in nearly 47,000 deaths, which is 6 times the number of opioid-related deaths in $1999 .^{2,3}$ In total, 218,000 Americans have died due to overdoses involving prescribed opioids between 1999 and 2017. ${ }^{3}$ Moreover, the number of deaths caused by prescribed opioid overdoses in 2017 was 5 times that in 1999. ${ }^{3}$ These statistics reflect the need for a shift in prescribing practices and perhaps in physician attitude toward opioid use. ${ }^{4}$ Although there is no straightforward or immediate solution, physicians can play a role in bringing it to and end by limiting unnecessary and excess opioid use in their patients.

Controlling access to illicit opioids is beyond a physician's scope of practice, but limiting the use of excess opioids in the community to prevent harm certainly falls within it. Over the last decade, the number of opioid prescriptions per 100 Americans peaked at 81.3 in $2012 .^{3}$ By 2017, it fell to 58.5 prescriptions per 100 people. $^{3}$ A potential reason for this drop in the number of opioid prescriptions is the widespread implementation and use of prescription drug-monitoring programs. More than $70 \%$ of all current prescription 
drug-monitoring programs in the United States were established between 2000 and $2015 .^{5}$ While it is clear that providers are striving to curb the number of opioid prescriptions written, there is still work to be done.

Physicians in surgical specialties are uniquely poised to combat this growing opioid crisis. One recent study of more than 35,000 patients found that opioid-naïve individuals who underwent minor and major surgeries developed persistent opioid use at rates between $5.9 \%$ and $6.5 \%$, with no difference between the 2 groups. ${ }^{6}$ Given the pain associated with orthopaedic procedures, it is common for patients to receive opioid analgesia in the perioperative period. In fact, a 2009 study of opioid use found that orthopaedic surgeons write $7.7 \%$ of all opioid prescriptions, the third most of all specialties. ${ }^{7}$ However, recently it has been shown that there is notable regional variability in opioid prescribing patterns for common orthopaedic procedures. ${ }^{8}$ Interestingly, research suggests that some nonsteroidal antiinflammatory drugs such as celecoxib can provide equal or greater pain control and tolerability compared with opioids following outpatient orthopaedic procedures. ${ }^{9}$ More recently, efforts to develop a multimodal approach to analgesia following major surgeries such as joint arthroplasty have proven successful: patients report decreased pain, experience shorter hospital stays, fewer complications, and are using fewer opioids postoperatively. ${ }^{10,11}$

Orthopaedic surgeons should strive to provide patients with adequate pain control without the risk of developing a life altering dependence on opioids. If patients can achieve postoperative analgesia with limited narcotics, orthopaedic surgeons may be able to further limit use of opioids during the perioperative period and avoid the many associated complications. Given the current public health crisis surrounding opioid abuse, there is a need to create reference points regarding prescribing practices for opioids following arthroscopic surgery. It is important for orthopaedic surgeons to understand what amount of opioid medication is generally necessary to manage postoperative pain following specific orthopaedic procedures to avoid overprescribing. The purposes of this study were to evaluate patient use of opioids following arthroscopic rotator cuff repair including number of days and number of pills when used in combination with nonopioid medications and to determine whether patients were satisfied with their pain management and if variables such as age, sex, body mass index (BMI), duration of symptoms, anticipation of postoperative pain, preoperative opioid consumption, size of the rotator cuff tear, or anxiety/depression affected pain management. We hypothesized that pain following arthroscopic rotator cuff repair could be managed with minimal opioid pain medication in combination with nonopioid pain medications, that the majority of patients would be satisfied with pain management, and that age, sex, BMI, and tear size would not affect their satisfaction with pain management.

\section{Methods}

Between January 2017 and October 2019, a consecutive cohort of 118 patients scheduled to undergo arthroscopic primary rotator cuff repair were prospectively enrolled in a Health Insurance Portability and Accountability Act of 1996 (HIPAA)-compliant institutional REDCap database for this study. All patients during the study period older than the age of 18 years who had a primary rotator cuff repair were included in the study. Exclusion criteria included incomplete preoperative and/or 2-week postoperative data. Patients were simultaneously enrolled in a HIPAA-compliant global registry, Surgical Outcome System (Arthrex, Naples, FL). This research study was reviewed by the Committee on Clinical Investigations and approved by the institutional review board (2011P002663 and 2016P002830). All patients provided an informed consent prior to participation in this study.

Questionnaires were completed in clinic electronically on an iPad at the preoperative and 2-week postoperative clinic appointments and were administered by a research assistant (K.D.G. or N.A.L.) The 2 -item Pain Self-Efficacy Questionnaire was administered to assess the confidence in one's ability to work and perform activities of daily life despite the pain. Screening for symptoms of anxiety and depression was conducted via the 2-item Patient Health Questionnaire. In the preoperative survey, patients reported any current opioid pain medication use and indicated their anticipated level of pain 48 hours following their upcoming surgery. History of current opioid use, duration of symptoms before surgery, surgical history, and other demographics were obtained.

Satisfaction with pain control was assessed using the Hospital Consumer Assessment of Healthcare Provider and Systems questionnaire. The Hospital Consumer Assessment of Healthcare Provider and Systems is a 27question patient-centric instrument designed to assess patient satisfaction with various aspects of hospital care, including pain management. ${ }^{12}$ In the postoperative survey, we used one question regarding pain control for our postoperative data collection: "In the time after surgery, how often was your pain well-controlled?" Answer choices were provided on a scale: always, usually, sometimes, and never. For analysis purposes, we established 2 groups for this question: "Always" and "Usually" were combined as the satisfied group, whereas "Sometimes" and "Never" were combined as the unsatisfied group. At the 2 -week visit, patients met with the research assistant and were asked to record both opioid and non-opioid medications taken during the 14-day postoperative period including type, dose, 
Table 1. Patient Demographics and Preoperative Assessments

\begin{tabular}{lc}
\hline \multicolumn{1}{c}{ Variable } & \\
\hline Total participants & 117 \\
Age, mean \pm SD & $55.8 \pm 9.8$ \\
Sex, female & $58(49.6 \%)$ \\
Race & $1(0.9 \%)$ \\
American Indian/Alaskan Native & $1(0.9 \%)$ \\
Asian & $2(1.7 \%)$ \\
Black or African American & $111(94.9 \%)$ \\
White & $2(1.7 \%)$ \\
Declined to specify & \\
Ethnicity & $5(4.3 \%)$ \\
Hispanic or Latino & $112(95.7 \%)$ \\
Not Hispanic or Latino & $27.9 \pm 5.1$ \\
BMI, mean \pm SD & $10(8.5 \%)$ \\
Current opioid use & $102(87.2 \%)$ \\
Previous surgery & $7(3-12)$ \\
Duration of symptoms, mo, median (Q1-Q3) & \\
Preoperative health assessment, mean \pm SD & $1.1 \pm 1.5$ \\
PHQ-2 & $6.6 \pm 3.1$ \\
PSEQ-2 & \\
Do you feel down, depressed, or hopeless? & $85(72.6 \%)$ \\
No & $32(27.4 \%)$ \\
Yes & $5.4 \pm 2.2$ \\
VAS, mean \pm SD & $6.9 \pm 2.2$ \\
Anticipated 48-h postoperative VAS, mean \pm SD & \\
\hline BMI, body mass index; PHQ-2, 2-item Patient Health Questionnaire; \\
PSEQ-2, 2-item Pain Self-Efficacy Questionnaire; SD, standard devi- \\
ation; VAS, visual analog pain scale.
\end{tabular}

and number of pills. Non-opioid pain medications were converted to $\mathrm{mg}$ /day to standardize the amount of pain medication patients required in the 2 -week postoperative period.

All patients underwent a primary arthroscopic rotator cuff repair (RCR) by a single surgeon (E.M.). Patients received an interscalene nerve block by the anesthesiologist before surgery and were discharged with a shoulder cryotherapy device and an abduction sling. All patients received prescriptions for 30 opioid pain pills ( 5 mg oxycodone), ibuprofen (800 mg), or naproxen and acetaminophen. Patients were instructed to alternate the ibuprofen/naproxen and acetaminophen and only use the opioid pain pills when needed.

\section{Statistical Analysis}

The primary outcome was the total number of opioid pills taken postoperatively. With a sample size of 117 , the study provided adequate precision for the primary outcome since the width of the $95 \%$ confidence interval was limited to 3.4 on each side. Secondary outcomes included visual analog scale (VAS) pain on postoperative day 2 , satisfaction with pain control, and total number and duration on an opioid pain medication within the first 2 weeks postoperatively. Categorical variables were summarized using frequency with percentage, and continuous variables were summarized using mean with standard deviation or median with interquartiles, whichever was more appropriate. In an exploratory data analysis, $\chi^{2}$ tests were used to compare preoperative opioid use and surgical characteristics between patients reporting always/usually and patients reporting sometimes/never for well-controlled pain, and the analysis of variance approach was used to examine the relationship between patient characteristics and amount/duration of narcotic pain medication usage. All analyses were conducted using SAS, version 9.4 (SAS Institute, Cary, NC).

\section{Results}

\section{Demographic Characteristics and Preoperative Patient-Reported Outcomes Measures}

In total, 118 patients were prospectively enrolled in this study, and 1 patient did not provide any follow-up data. The remaining 117 patients (58 female and 59 male) were included in the study with a mean age of $55.8 \pm 9.8$ years (range, 21-76 years), mean BMI $27.9 \pm 5.1$, and median duration of symptoms of 7 months (interquartiles, 3-12 months). One patient was excluded for incomplete postoperative data. At the preoperative clinic visit, the mean VAS pain score was $5.4 \pm 2.2$. 107 patients $(91.5 \%)$ were opioid-naïve before surgery and 10 patients $(8.5 \%)$ were taking opioid pain medication preoperatively. Before surgery, the mean anticipated VAS at 48 hours postoperatively was $6.9 \pm 2.2$, indicating an anticipation of pain greater than their preoperative pain (Table 1). Surgical characteristics including biceps procedures, numbers of tendons torn, tear thickness, and Cofield classification of tear size are all reported in Table 2.

\section{Postoperative Pain Medication Requirement}

A multimodal pain management approach that included a combination of non-opioid (ibuprofen or naproxen and acetaminophen) and opioid medication and a cryotherapy device was provided to all patients. At the 2-week postoperative visit, 109 (93.2\%) patients reported taking opioid pain medication after surgery whereas $8(6.8 \%)$ patients did not require any opioid pain medication following surgery (Table 3 ). Of the

Table 2. Diagnostic and Surgical Characteristics

\begin{tabular}{lc}
\hline \multicolumn{1}{c}{ Variable } & $\mathrm{N}(\%)$ \\
\hline Biceps procedure & \\
$\quad$ No & $93(79.5)$ \\
Yes & $24(21.5)$ \\
Number of tendons involved & \\
1 & $65(55.5)$ \\
2 & $38(32.5)$ \\
3 & $14(12.0)$ \\
Tear size & \\
Small $(<1 \mathrm{~cm})$ & $21(17.9)$ \\
Medium $(1-3 \mathrm{~cm})$ & $75(64.1)$ \\
Large $(3-5 \mathrm{~cm})$ & $17(14.5)$ \\
Massive $(>5 \mathrm{~cm})$ & $4(3.4)$ \\
\hline
\end{tabular}


Table 3. Primary Postoperative Outcomes

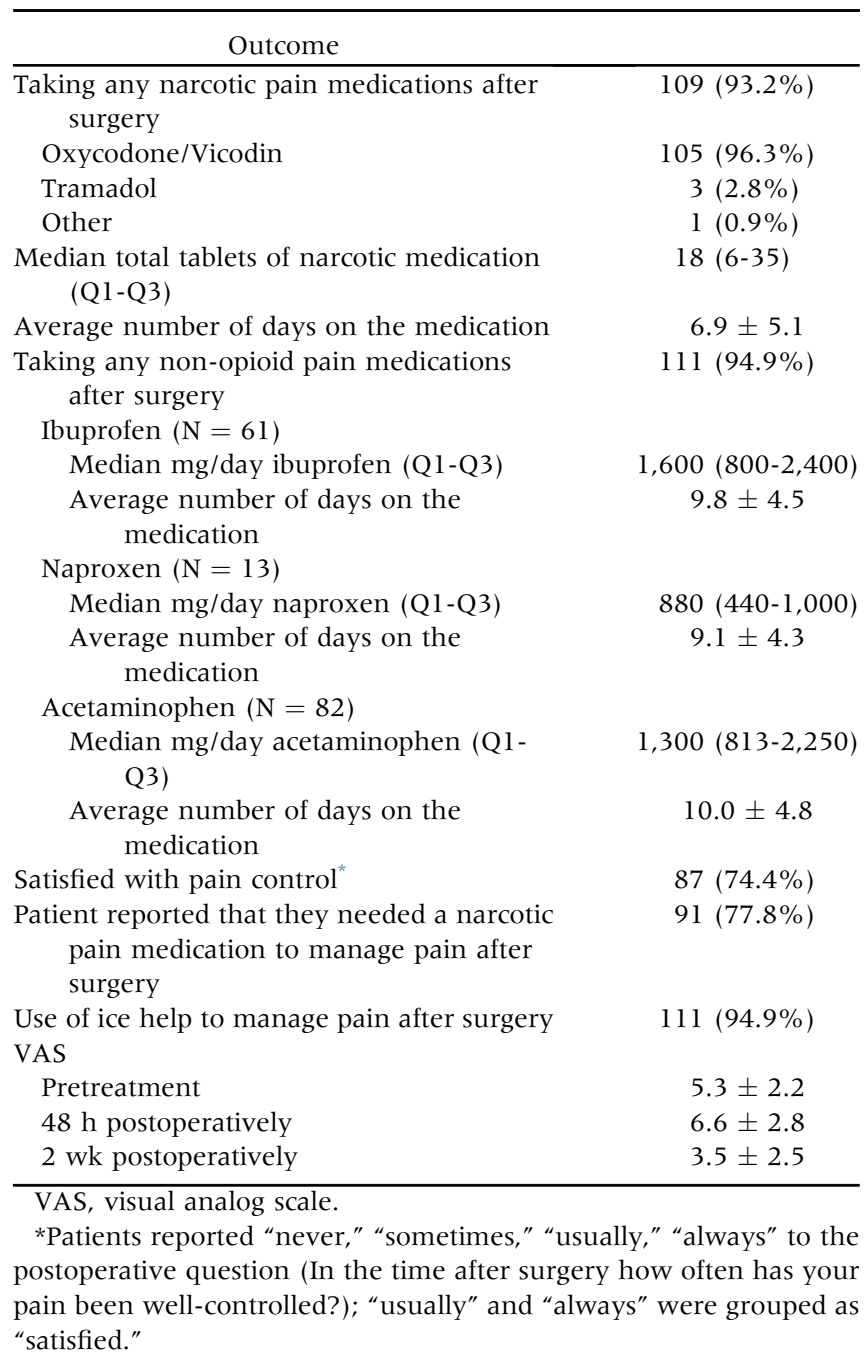

patients who took opioid pain medication following surgery, the median cumulative opioid tablet intake was 18 (interquartiles, 6-35 tablets), approximately 135-mg morphine equivalents, and the mean duration of opioid consumption was $6.9 \pm 5.1$ days and $65 \%$ of patients did not take opioid pain medications for longer than 7 days (Table 3).

All patients were instructed to use non-opioid pain medications to manage their pain following surgery, using the opioid medication only when needed. In total, $94.9 \%$ of patients reported taking either ibuprofen/ naproxen or acetaminophen for postoperative pain. Of the patients who reported taking ibuprofen or naproxen, the average number of days they were on the medication were $9.8 \pm 4.5$ and $9.1 \pm 4.3$ days, respectively. Patients using ibuprofen reported taking a median of $1600 \mathrm{mg}$ (interquartiles, 800-2400 mg), and patients using naproxen reported taking a median of $880 \mathrm{mg}$ (interquartiles, 440-1000 mg) per day (Table 3). Lastly, patients who took acetaminophen reported taking a median of $1300 \mathrm{mg}$ (interquartiles, 813-2250 $\mathrm{mg}$ ) per day over a period of $10 \pm 4.8$ days total (Table 3).

\section{Satisfaction With Postoperative Pain Management}

At the 2 -week postoperative time point $74.4 \%$ of patients reported their pain being "always or usually" well-controlled. In total, $77.8 \%$ of patients reported yes to feeling like "they needed a narcotic pain medication to manage pain after surgery," and $94.9 \%$ of patients reported that the use of ice helped to reduce pain after surgery (Table 3 ). Patients reported a mean VAS pain score of $6.6 \pm 2.8$ in the 48 hours following surgery and a mean VAS pain score was $3.5 \pm 2.5$ at the 2 -week postoperative visit (Table 3). Age, sex, BMI, duration of symptoms, anticipation of postoperative pain, preoperative opioid use and surgical characteristics were not significant predictors of patient satisfaction or the number of opioid tablets or duration of use. Surgical characteristics were not significantly associated with either amount or duration of narcotic pain medication usage (Table 4).

\section{Discussion}

The results of this study demonstrate that a minimum number of opioid tablets in conjunction with nonopioid pain medication can successfully provide satisfactory pain control following arthroscopic RCR. Specifically, this prospective study defined a mean opioid requirement of 18 opioid pain pills (135 mg morphine equivalents) over a mean duration of $6.9 \pm$ 5.1 days for patients undergoing a primary arthroscopic RCR. Preoperative measurement of anticipation of pain at 48 hours postoperatively was greater than preoperative pain $(6.9 \pm 2.2$ vs $5.4 \pm 2.2$, respectively). It is important to recognize if patients are achieving a minimal clinically important difference (MCID) or patient acceptable symptom state (PASS) postoperatively rather than just statistical significance. ${ }^{13}$ Tashjian et al. ${ }^{14}$ demonstrated that a VAS improvement in patients treated for rotator cuff disease as a MCID of 1.4 points $(P=.0255)$ and a PASS of 3 points $(95 \%$ confidence interval 22.69-37.31) on a 10-point VAS measuring pain. In the current study, at 48 hours postoperatively, $14.5 \%$ of patients achieved a MCID of 1.4 points' and $8.5 \%$ a PASS of 3 points' improvement. At the 2-week postoperative time point, $54.7 \%$ achieved an MCID of 1.4 points' improvement and 35.0\% achieved a PASS of 3 points' improvement.

Using the described multimodal pain regiment, $74.4 \%$ of patients were satisfied with their postoperative pain management. Differences in age, sex, BMI, duration of symptoms, anticipation of postoperative pain, preoperative 2-item Patient Health Questionnaire, 2-item Pain Self-Efficacy Questionnaire, current opioid use, and surgical characteristics had no 
Table 4. Predictors of Satisfaction with Postoperative Pain Control, Amount and Duration of Opioid Use for Postoperative Pain Control

\begin{tabular}{|c|c|c|c|c|c|c|}
\hline \multirow[b]{2}{*}{ Characteristic } & \multirow{2}{*}{$\frac{\text { Satisfaction* }}{\mathrm{N}(\%)}$} & \multirow[b]{2}{*}{$P$ Value } & \multirow{2}{*}{$\begin{array}{c}\text { Total Number of Narcotic } \\
\text { Tablets } \\
\text { Mean } \pm \text { SD }\end{array}$} & \multirow[b]{2}{*}{$P$ Value } & Duration of Use & \multirow[b]{2}{*}{$P$ Value } \\
\hline & & & & & Mean \pm SD & \\
\hline Age, y & & .32 & & .14 & & .1 \\
\hline$<60$ & $58(77.3)$ & & $22.4 \pm 19.9$ & & $7.0 \pm 5.5$ & \\
\hline$\geq 60$ & $29(69.0)$ & & $17.0 \pm 15.3$ & & $5.4 \pm 4.7$ & \\
\hline Sex & & .43 & & .22 & & .69 \\
\hline BMI & & .47 & & .08 & & .32 \\
\hline$<30$ & $64(76.2)$ & & $18.5 \pm 17.5$ & & $6.1 \pm 5.3$ & \\
\hline$\geq 30$ & $23(69.7)$ & & $25.2 \pm 20.0$ & & $7.2 \pm 5.1$ & \\
\hline \multicolumn{2}{|c|}{ Symptom duration, mo } & .5 & & .76 & & .93 \\
\hline$<6$ & $35(77.8)$ & & $19.8 \pm 17.7$ & & $6.4 \pm 5.1$ & \\
\hline$\geq 6$ & $52(72.2)$ & & $20.9 \pm 19.0$ & & $6.5 \pm 5.4$ & \\
\hline$<5$ & $11(64.7)$ & & $15.8 \pm 13.6$ & & $5.6 \pm 4.5$ & \\
\hline$\geq 5$ & $76(76.0)$ & & $21.3 \pm 19.1$ & & $6.6 \pm 5.4$ & \\
\hline \multicolumn{2}{|c|}{ Preoperative opioid use } & .28 & & .53 & & .24 \\
\hline No & $81(75.7)$ & & $20.1 \pm 18.4$ & & $6.3 \pm 5.2$ & \\
\hline Yes & $6(60.0)$ & & $24.0 \pm 19.6$ & & $8.3 \pm 5.4$ & \\
\hline \multicolumn{2}{|c|}{ Number of tendons involved } & .61 & & .36 & & .56 \\
\hline 1 & $49(71)$ & & $22.1 \pm 19.7$ & & $6.7 \pm 5.2$ & \\
\hline 2 & $27(71.1)$ & & $16.9 \pm 16.0$ & & $5.7 \pm 5.4$ & \\
\hline 3 & $11(78.6)$ & & $22.7 \pm 18.6$ & & $7.2 \pm 5.0$ & \\
\hline \multicolumn{2}{|c|}{ Tear size (Cofield classification) } & .39 & & .52 & & .59 \\
\hline Small & $18(85.7)$ & & $15.7 \pm 16.5$ & & $5.5 \pm 5.2$ & \\
\hline Medium & $52(69.3)$ & & $22.3 \pm 19.2$ & & $6.9 \pm 5.3$ & \\
\hline
\end{tabular}

BMI, body mass index; SD, standard deviation.

effect on, or association with, satisfaction with pain management postoperatively.

It is important to optimize opioid pain medication prescriptions to limit surplus pills and to limit the risk of patients developing prolonged opioid use or opioid use disorder. In a recent 2019 study, Gil et al. ${ }^{15}$ found that $8.3 \%$ of patients who underwent an arthroscopic shoulder procedure developed prolonged opioid use, defined as greater than or equal to 1 prescription being filled between 91 and 180 days after the initial surgical event. ${ }^{15}$ Gil et al. also found that greater opioid consumption ( $\geq 743$ oral morphine equivalents; or at least 149 5-mg hydrocodone) during the perioperative period increased the risk for prolonged opioid use. Our current prospective study demonstrated that when combined with non-opioid medications, a median of 18 opioid pills (135 oral morphine equivalents) over an average of approximately 1 week was used for postoperative RCR pain control, suggesting that this amount of pain medication carries limited risk for developing dependence or prolonged opioid use. These results are also consistent with another study that showed $77 \%$ of patients took fewer than 15 opioid pills when paired with an interscalene nerve block and with non-opioid medications were implemented. ${ }^{16}$ Recent studies have demonstrated that preoperative opioid use in arthroscopic RCR can lead to increased postoperative opioid requirements. ${ }^{17,18}$ Further, patients who filled opioid prescriptions preoperatively were 7.45 times more likely to fill opioid prescriptions postoperatively. ${ }^{17}$ The results of this current study, however, did not demonstrate an association between preoperative opioid use $(8.5 \%)$ and increased postoperative opioid consumption $P=.53$ and duration $P=.24$; however, no meaningful conclusions can be made, as there were only a small number of preoperative opioid users.

Sabatino et al. ${ }^{19}$ reviewed prescribing patterns after common orthopaedic procedures and found that an average of 80 pills (oxycodone 5 -mg equivalent) were prescribed on discharge following arthroscopic RCR and $61 \%$ of the patients reported having unused opioid medication. Taking into account previous literature and 
the results of the current study, which shows that the mean number of pills consumed in our patient cohort is approximately one-fourth the amount of pills regularly prescribed after this procedure, it is clear there is significant room to reduce the total amount of pills prescribed while maintaining satisfactory pain control for the patient.

Many studies have looked at risk factors for developing prolonged opioid use or dependence following orthopaedic procedures. Recent literature has shown that preoperative pain is a strong predictor for postoperative pain following RCR. ${ }^{20}$ This study also suggested that alongside the association of severity and frequency of preoperative pain with postoperative pain, other factors such as smaller tear size, young age, female sex, and worker's compensation status were associated with 6-week postoperative pain after RCR. ${ }^{20}$ Leroux et al. ${ }^{21}$ reviewed 79,287 patients who underwent elective shoulder surgery and found that history of alcohol abuse, depression, or anxiety and female sex all had the most significant influence on risk of developing long-term opioid use. Several studies have also demonstrated that opioid use before surgery resulted in both increased amount and duration of opioid use postoperatively. ${ }^{4,17,18}$ Another study showed that opioid-naïve patients demonstrate significantly better patient-reported outcomes following RCR, suggesting that orthopaedic surgeons must factor in a patients preoperative opioid use and educate patients regarding pain expectations in the immediate postoperative period. ${ }^{22}$ Recently, Farley et al. ${ }^{23}$ showed that preoperative opioid use and increased dosage was a risk factor for postoperative complications and revision surgery. In our cohort, only $8.5 \%$ of patients used opioids preoperatively, and this had no association with postoperative pain management satisfaction. Further, $74.4 \%$ of patients were satisfied with their pain management and variables such as age, sex, BMI, duration of symptoms, anticipation of postoperative pain, or surgical characteristics such as tear size, thickness, or tendons involved did not affect overall satisfaction with pain management.

A pain regimen that uses multimodal analgesia following arthroscopic RCR has the potential to reduce overall opioid consumption. Preoperative pain education, interscalene nerve block, intraoperative injection, cryotherapy, and nonopioid pain medications can all contribute to a decreased reliance on opioid pain medication. ${ }^{24}$ In our study, all patients received preoperative counseling by their surgeon on pain expectations, an interscalene nerve block, and a shoulder cryotherapy device. Patients were instructed to take ibuprofen or naproxen regularly, alternating with acetaminophen and were encouraged to use the opioids only when needed. Of the 117 patients, 111 (94.9\%) reported that the use of cryotherapy/ice helped reduce their pain after surgery. On average, patients took the nonopioid pain medications for a longer duration (approximately 9-10 days postoperative) than the opioid pain medication $(6.9 \pm 5.1$ days $)$.

Overall, orthopaedic surgeons have a responsibility to provide a safe and effective postoperative pain management plan for all patients undergoing arthroscopic RCR and knowledge of average postoperative pain medication use and postoperative pain levels is essential to developing a successful pain management regimen.

\section{Limitations}

All data regarding amount and duration of opioid and non-opioid medication consumption are patientreported and subject to recall bias. Recall biases were minimized because this is a prospective study, and the follow-up data were collected only 1 day and 2 weeks after surgery. The early postoperative follow-up period is intentional with the prospective design of this study; however, it is not predictive of pain in longer-term follow-up and overall outcomes of arthroscopic RCR. There was a loss to follow-up of 1 patient as a result of incomplete postoperative data. All 117 other patients enrolled in the study had complete data. Another limitation of this study includes selection bias and the potential lack of generalizability since all data was collected from patients within an institution from a single sports medicine surgeon. Although this is a limitation, there is a potential benefit in that it limited variability in preoperative planning, setting expectations, surgical technique, and postoperative management.

\section{Conclusions}

Following arthroscopic RCR, patients can achieve satisfactory pain control using a multimodal approach with a median of 18 opioid pills (range, 6-35 pills) over $6.9 \pm 5.1$ days when used in combination with nonopioid pain medications. Overall, $74.4 \%$ of patients were satisfied with pain management.

\section{References}

1. LaPietra AM, Motov S. A country in crisis: Opioid sparing solutions for acute pain management. Mo Med 2019;116: 140-145.

2. Centers for Disease Control and Prevention. Annual Surveillance Report of Drug-Related Risks and Outcomes. Surveillance Special Report. Atlanta, GA: Centers for Disease Control and Prevention, U.S. Department of Health and Human Services, August 31, 2018.

3. Centers for Disease Control and Prevention. Opioid Overdose. Centers for Disease Control and Prevention, U. S. Department of Health and Human Services, https:// www.cdc.gov/drugoverdose/data/analysis.html. Accessed January 25, 2021.

4. Chapman CG, Hudspeth J, Floyd SB, Carnahan R, Thigpen CA, Kissenberth MJ. Increased opioid use following rotator cuff repair associated with prior opioid 
use and surgeon prescription patterns. J Shoulder Elbow Surg 2020;29:S115-S125.

5. Holmgren AJ, Botelho A, Brandt AM. A history of prescription drug monitoring programs in the United States: Political appeal and public health efficacy. Am J Public Health 2020;1 10:1191-1197.

6. Brummett CM, Waljee JF, Goesling J, et al. New persistent opioid use after minor and major surgical procedures in US adults. JAMA Surg 2017;152:e170504.

7. Volkow ND, McLellan TA, Cotto JH, Karithanom M, Weiss SR. Characteristics of opioid prescriptions in 2009. JAMA 2011;305:1299-1301.

8. Traven SA, Brinton DL, Woolf SK, Leddy LR, Slone HS. Significant variability in opioid prescribing practices after common orthopaedic procedures. Arthroscopy 2021;37:e77.

9. Gimbel JS, Brugger A, Zhao W, Verburg KM, Geis GS. Efficacy and tolerability of celecoxib versus hydrocodone/ acetaminophen in the treatment of pain after ambulatory orthopedic surgery in adults. Clin Ther 2001;23:228-241.

10. McLaughlin DC, Cheah JW, Aleshi P, Zhang AL, Ma CB, Feeley BT. Multimodal analgesia decreases opioid consumption after shoulder arthroplasty: A prospective cohort study. J Shoulder Elbow Surg 2018;27:686-691.

11. Jildeh TR, Moutzouros V, Khalil LS, et al. Can we eliminate opioids after common orthopedic sports procedures? A novel multimodal pain protocol. Arthroscopy $2021 ; 37:$ e34.

12. HCAHPS Fact Sheet (CAHPS Hospital Survey). Centers for Medicare \& Medicaid Services, https://hcahpsonline.org/ globalassets/hcahps/facts/hcahps_fact_sheet_november_ 2017.pdf. Accessed August 16, 2021.

13. Harris JD, Brand JC, Cote MP, Faucett SC, Dhawan A. Research pearls: The significance of statistics and perils of pooling. Part 1: Clinical versus statistical significance. Arthroscopy 2017;33:1102-1112.

14. Tashjian RZ, Deloach J, Porucznik CA, Powell AP. Minimal clinically important differences (MCID) and patient acceptable symptomatic state (PASS) for visual analog scales (VAS) measuring pain in patients treated for rotator cuff disease. J Shoulder Elbow Surg 2009;18:927-932.
15. Gil JA, Gunaseelan V, DeFroda SF, Brummett CM, Bedi A, Waljee JF. Risk of prolonged opioid use among opioid-naïve patients after common shoulder arthroscopy procedures. Am J Sports Med 2019;47:1043-1050.

16. Mandava NK, Sethi PM, Routman HD, Liddy N, Haidamous G, Denard PJ. Opioid requirement after rotator cuff repair is low with a multimodal approach to pain. J Shoulder Elbow Surg 2021;30:e399-e408.

17. Westermann RW, Anthony CA, Bedard N, et al. Opioid consumption after rotator cuff repair. Arthroscopy 2017;33: 1467-1472.

18. Williams BT, Redlich NJ, Mickschl DJ, Grindel SI. Influence of preoperative opioid use on postoperative outcomes and opioid use after arthroscopic rotator cuff repair. J Shoulder Elbow Surg 2019;28:453-460.

19. Sabatino MJ, Kunkel ST, Ramkumar DB, Keeney BJ, Jevsevar DS. Excess opioid medication and variation in prescribing patterns following common orthopaedic procedures. J Bone Joint Surg Am 2018;100:180-188.

20. Rizvi SMT, Bishop M, Lam PH, Murrell GAC. Factors predicting frequency and severity of postoperative pain after arthroscopic rotator cuff repair surgery. Am J Sports Med 2021;49:146-153.

21. Leroux TS, Saltzman BM, Sumner SA, et al. Elective shoulder surgery in the opioid naïve: rates of and risk factors for long-term postoperative opioid use. Am J Sports Med 2019;47:1051-1056.

22. Sabesan VJ, Petersen-Fitts GR, Sweet MC, Katz DL, Lima DJL, Whaley JD. The impact of preoperative opioid use on outcomes after arthroscopic rotator cuff repair. JSES Open Access 2018;2:155-158.

23. Farley KX, Wilson JM, Spencer CC, et al. Preoperative opioid use is a risk factor for revision surgery, complications, and increased resource utilization after arthroscopic rotator cuff repair. Am J Sports Med 2020;48:3339-3346.

24. Sanchez-Sotelo J. Multimodal pain management may successfully replace opioids in arthroscopic rotator cuff repair. Healio. https://www.healio.com/news/orthopedics/ 20200501/multimodal-pain-management-may-successfullyreplace-opioids-in-arthroscopic-rotator-cuff-repair. Accessed February 12, 2021. 